\title{
Antifreeze Proteins: An Unusual Tale of Structural Evolution, Hydration and Function
}

\author{
SANDIPAN CHAKRABORTY and BIMAN JANA* \\ School of Chemical Sciences, Indian Association for the Cultivation of Science, Jadavpur, \\ Kolkata 700 032, India
}

(Received on 18 September 2018; Revised on 14 November 2018; Accepted on 14 November 2018)

\begin{abstract}
Organisms living in extreme cold conditions find a unique solution for cold adaptation. They produce a group of proteins, called antifreeze proteins (AFPs) which cause depression of freezing point in a non-colligative manner. AFPs are unusual in every sense, i.e., their evolution, hydration and function. Evolutionary analysis reveals that AFPs do not emerge from a common ancestor; rather their evolution is independent and closely correlated to the Antarctic glaciation events. AFPs exert antifreeze activity by adsorption-inhibition mechanism. According to the model, ice can grow in the region between two bound AFPs, which results in the generation of a curved growing ice front. As a result, the radius of curvature decreases with further growth of the ice front and ultimately halts at the limit of critical radius. Main assumption of the model is that AFPs irreversibly bind to the ice surface, which is also validated by recent microfluidic experiments. Initially hydrogen bond mediated ice binding has been proposed, but experimental mutation data invalidated the hypothesis. A surface complementarity driven ice adsorption mechanism has been lately proposed, however, it is inadequate to explain the irreversible binding. Mechanism of ice recognition by AFP still remains elusive. Recently, AFPs have been shown to induce water ordering and we have proposed that the ordered hydration water adopts clathrate-like structure, which effectively adsorbs AFP on ice. Hydrophobic hydration induces clathrate formation around the ice binding surface. The model is highly promising and qualitatively explains mutational data and binding plane specificity data from ice-etching experiments.
\end{abstract}

Keywords: Antifreeze Proteins; Evolution; Hydration; Ice Recognition; Clathrate

\section{Introduction}

In the early $19^{\text {th }}$ century, the survival of organisms in the extreme cold condition during Antarctic winter was astonoshing. During 1960s, it was realized that some special elements exist that help these organisms in cold-acclimatization (Gordon et al., 1962). Pure water and ice are in equilibrium at $0{ }^{\circ} \mathrm{C}$, the freezing/ melting point. Addition of solutes shifts this equilibrium. Sea water in Arctic region contains large concentrations of salt and other solutes that cause depression of freezing point, however, in a colligative manner. The freezing point of sea water is $\sim-1.9^{\circ} \mathrm{C}$. However, the plasma solute concentration of teleost fish is found to be much lower, which results in the depression of freezing point up to $-0.7^{\circ} \mathrm{C}$ ( $\mathrm{Jia}$ and Davies, 2002). Therefore, it is expected that Antarctic fishes should die in contact with the cold sea water.
However, organisms still survive in extreme cold condition. Arthur L. DeVries and Donald E. Wohlschlag first identified the surprizing element from the blood of a marine fish. A glycoprotein was identified that accounted for $\sim 30 \%$ depression of freezing point in fish serum (DeVries and Wohlschlag, 1969). Later, this class of proteins was classified as antifreeze glycoproteins (AFGPs) to differentiate them from antifreeze proteins/peptides (AFPs). With time, more mysteries on antifreeze protein started to unfold. The protein is highly surprising to both structural biologists and evolutionary biologists as its functions and evolution appear to be very complex phenomenon and till date remains highly debatable (Cheng, 1998). During late $19^{\text {th }}$ and early $20^{\text {th }}$ century, there were renaissance in AFP research. Different AFPs from different organisms were identified and

*Author for Correspondence: E-mail: pcbj@iacs.res.in 
their structures were solved using crystallography and nuclear magnetic resonance spectroscopy. Now it is known that antifreeze proteins are abundant in fishes, insects, plants, bacteria and fungi in the Antarctic regions (Devries, 1971; Duman, 2001; Ewart et al., 1999; Griffith et al., 1997; Jia and Davies, 2002). Even within fish, multiple varieties of AFPs are produced. Fish AFPs are in general less active in terms of thermal hysteresis $\left(\sim 1-1.5^{\circ} \mathrm{C}\right)$, whereas insect AFPs can cause thermal hysteresis up to $-8^{\circ} \mathrm{C}$, therefore termed as hyperactive (Graether et al., 2000; Hanada et al., 2014; Scotter et al., 2006). The biological significance of this fact is that during Antarctic winter, atmospheric temperature is colder compared to the sea water, thus insects are facing more extreme cold environments. Therefore, insect AFPs were evolved in such a way that this becomes more potent in terms of thermal hysteresis. Additionally, different classes of AFPs do not bear any structural similarities; even they do not belong to a particular fold which indicates a complex evolutionary origin. Recent studies revealed that AFPs, in general, possess the ability to control water dynamics around its ice binding site (Nutt and Smith, 2008; Smolin and Daggett, 2008), even long range modulation of water dynamics by AFP also has been reported (Mallajosyula et al., 2014; Meister et al., 2013). However, due to the difficulty in characterizing AFP on the ice surface, the mechanism of ice recognition by AFPs still remains debatable. Many competing hypotheses have been proposed, but none of them conclusively explain experimental anomalies and not unanimously applicable to all classes of AFPs. Clathrate mediated ice adsorption is the most recent proposal that qualitatively explains mutational data and validates experimentally observed binding plane specificity data (Chakraborty and Jana, 2017b; Garnham et al., 2011). This review discusses different dimensions of AFP researches in sufficient details.

\section{Structural Diversity of Antifreeze Proteins: Effect of Independent Evolutionary Origin}

AFPs are generally found in organisms of the Polar regions. Although their abundance is confined within the Polar regions, structurally they are diverse. Eventually, AFPs from different groups are topologically different, as well as their activities also differ greatly. The large variations in structure and activity signify their independent evolutionary pattern. Fish AFPs are generally classified in five sub-types: type I, II, III, IV and antifreeze glycoproteins (AFGPs) (Jia and Davies, 2002). The rationale behind the number of different types of fish AFPs is actually correlated to their order of discovery. Representative structures from different classes of AFPs are shown in Fig. 1. In addition, two types of AFPs are found in polar insects, beetle (Rhagium inquisitor, Tenebrio molitor) and moth (Choristoneura fumiferana). Later, AFPs were also identified from freeze-tolerant plants, fungi, diatoms and even in bacteria (Kim et al., 2017).

Type I AFPs are in general a single straight helix. Sequence analysis reveals repetition of a sequence motif of 11 residues "TXXD/NXXXXXXX", where $X$ can be any residue but in most of cases it is ALA (Kun and Mastai, 2007). Structural analysis shows remarkable periodicity of hydrogen bonding residues THR and ASN/ASP in one side of the protein, which presumably is the ice binding site (IBS). Recently, an unusual structure of a type I AFP was reported which is four helix bundle composed of two dimers with a hydrate core (Sun et al., 2014). Detailed thermodynamic analysis suggests that the functionally active state of the protein is probably the cold denatured state (Parui and Jana, 2018). Evolutionary precursor of type I AFP is unknown, however, they are not derived from any other type of AFP.

Type II AFPs are cysteine-rich globular AFPs. Interestingly, this type of AFP is found in three different fish families (sea raven, smelt and Arctic herring) that are very divergent (Cheng, 1998). Sequence analysis reveals that they are homologous to $\mathrm{Ca}^{+2}$ dependent carbohydrate binding protein from lectin family (Liu et al., 2007b). Multiple sequence analysis followed by phylogenetic analysis of different type II AFPs along with several carbohydrate binding lectins reveal that $\mathrm{Ca}^{2+}$-dependent and independent type II AFPs form separate clade among lectins which indicate that they might evolve from a common ancestor. Fish lectins bear all the required amino acids for binding to two $\mathrm{Ca}^{2+}$ ions near the carbohydrate-binding site. However, $\mathrm{Ca}^{2+}$-dependent type II AFPs contains residues required to bind only one $\mathrm{Ca}^{2+}$ ion. In $\mathrm{Ca}^{2+}$ independent type II AFPs, most of the ion chelating residues are lost. Authors proposed that type II AFPs are generated due to gene duplication and subsequent functionalization of the same ancestor teleost fish lectin (Liu et al., 2007b). 


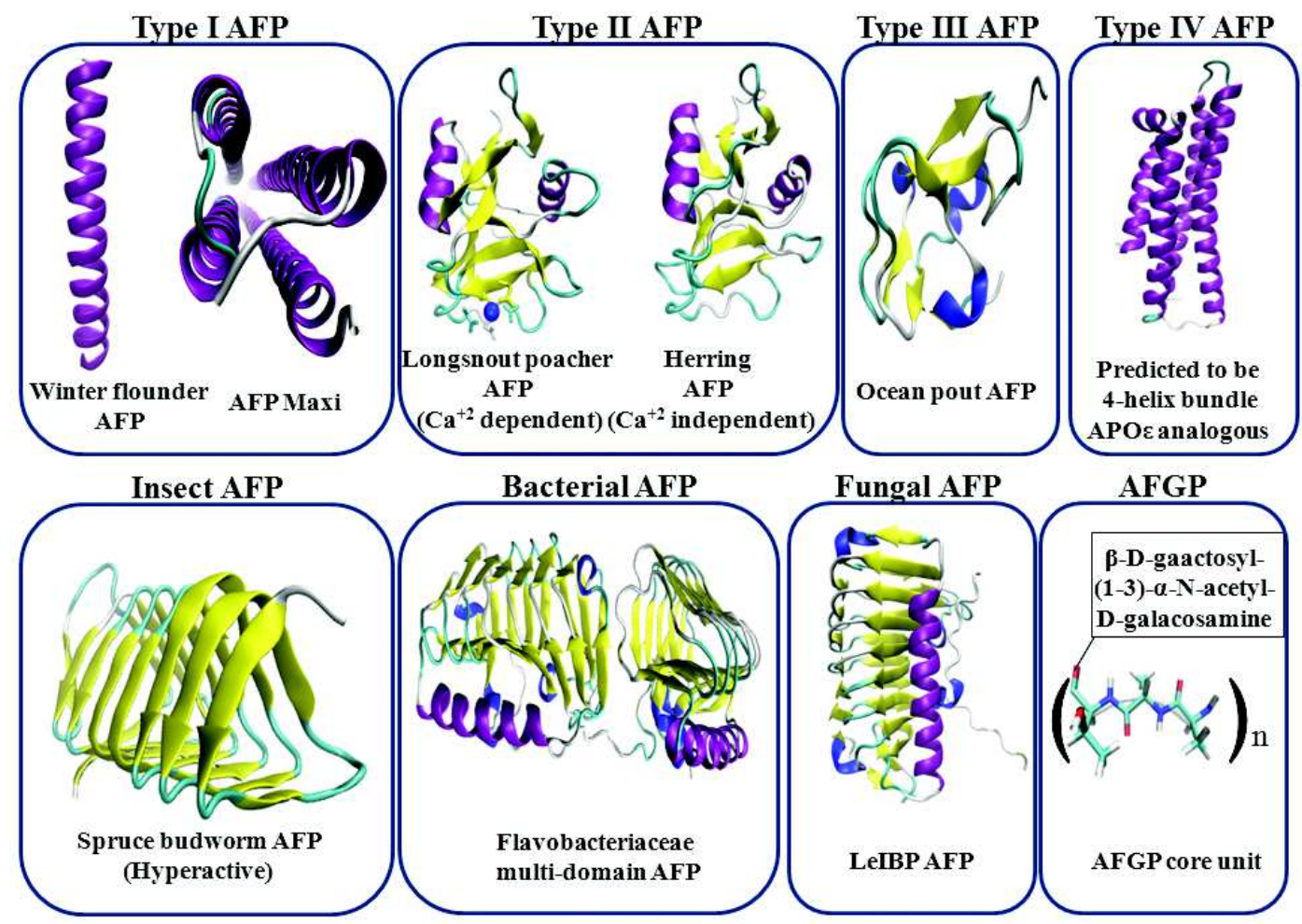

Fig. 1: Structures of different classes of AFPs are shown here. AFPs are rendered in ribbon representation and coloured according to their secondary structures

Type III AFPs are globular proteins devoid of any sequence repeat. Sequence analysis reveals high similarity between fish type III AFPs and the Cterminal region of mammalian sialic acid synthase (SAS) (Baardsnes and Davies, 2001). Type III AFPs and human SAS are $40 \%$ identical; the identity is particularly high on the ice binding site (IBS) (44\%).

Type IV AFPs were discovered much later. The longhorn sculpin type IV AFP has been predicted to be an anti-parallel helix bundle. However, sequence analysis identifies homology with apolipoprotein protein superfamily (Deng et al., 1998). Apparently, type IV AFPs do not contain sequence repeats. Vertebrate apolipoproteins contain 22 residue tandem repeats. Refinement of the sequence alignments between longhorn sculpin type IV AFP sequence and vertebrate apolipoproteins revealed 22 residue repeats within the region 28-104 of the AFP (Cheng, 1998).

AFGPs on the other hand are mostly unstructured in solution. There are eight different isoforms of AFGPs with different sizes. However, almost all the isoforms have similar sequence motifs.
Each motif is composed of ALA-ALA-THR repeat where the disaccharide beta-D-galactosyl-(1->3)alpha-N-acetyl-D-galactosamine moiety is attached to the hydroxyl group of the THR residue (Chen et al., 1997). In case of AFGP isoforms 6-8, the ALA in the repeats is sometimes replaced by PRO. Initially in the translated protein, all the isoforms are linked by conserved three-residue spacers (mostly LEU/PHEILE/ASN-PHE) and during post-translational modifications these spacers are removed to generate of multiple copies of mature AFGP per gene. The organization of AFGP gene is composed of two exons and a single intron. The small exon encodes the signal peptide and the large one encodes the AFGP polypeptides. AFGPs are found in two different divergent fish species, Antarctic notothenioid and in several Arctic and north Atlantic cods. In Cod AFGPs, often the THR residue of the tri-peptide sequence repeat is replaced by ARG. Evolution of AFGPs is puzzling and yet to understand completely. The mystery lies in the fact that two geographically and phylogenetically distantly related fish species produce near-identical AFGPs. Initial studies suggest that the 
two groups evolved their AFGPs independently. Database similarity searches reveal AFGPs genes of notothenioid shows $>70 \%$ identity with the 3 ' end of a trypsinogen cDNA from a pleuronectiform teleost, Pleuronectes platessa, which clearly suggest that notothenoid AFGPs and fish tripsinogens are evolutionary related (Chen et al., 1997). However, it is astonishing that two similar genes encode two different proteins that bear almost no resemblance in their structure and function. Detailed study reveals that the front and tail of trypsinogen genes are primarily recruited to form the AFGP gene family. Those regions in the AFGP genes share 93-96\% sequence identity with the trypsinogen gene. This region of the ancestral trypsinogen gene undergoes several events of nucleotide insertion, deletion and duplication to produce precursor AFGP genes, along with shedding of the trypsin coding sequence of the gene (Exon 2) (Chen et al., 1997). These event leads to produce two highly different proteins with very divergent structure and function. The Arctic cod AFGP genes share very little homology with the trypsinogen which indicates that they must have evolved from a different genomic locus. Thus, the evidence suggests convergent evolution of AFGPs from northern cods and Antarctic notothenioids.

Insect antifreeze proteins, on the other hand, draw much attention in recent years due to the fact that among all classes of AFPs, this group of AFP is hyperactive. The thermal hysteresis activity ( $\mathrm{TH}$ activity) exhibited by insect AFPs is approximately 10-100 times higher, compared to the equimolar fish AFPs (Kim et al., 2017). Different insect AFPs exhibit TH activity in the order of 4-6 degrees. AFP isolated from the insect longhorn beetle Rhagium mordax shows TH activity up to 8 degree, the most potent AFP known till date (Buch and Ramløv, 2017). Crystal structures of insect AFP from spruce budworm (Choristoneura fumiferana) and the beetle Tenebrio molitor were first solved. Insect AFP from spruce budworm is a $\beta$-helix with a triangular cross-section and each side is composed of parallel $\beta$-sheets, on the other hand Tenebrio molitor AFP is shaped like a slightly flattened cylinder of $32 \AA$ length with a pseudo-rectangular cross section. However, the IBS of both the insect AFPs share very similar topology with an extremely flat, rigid $\beta$-sheeted surface (Graether et al., 2000). In terms of sequence, both the insect AFPs share 70\% sequence identity and rich in CYS, SER and THR residues. The IBS of both the AFPs bears the regular array of TXT motif (Graether et al., 2000). However, the recently solved crystal structure of another highly active AFP from Rhagium inquisitor reveals a different fold (Hakim et al., 2013). The AFP exhibits solenoid-like architecture. The IBS is extremely flat and contains perfectly oriented extensive array of THR residues. More recently, new AFPs from this class have been identified. Recently, solved structure of AFP from adult midges shows a repeating unit of X-X-C-X-G-X-Y$\mathrm{C}-\mathrm{X}-\mathrm{G}$ with disulphide bonds stabilizing the left-handed solenoid-like structure. The AFP is moderately active and its IBS is constituted of a series of perfectly aligned tyrosine residues.

Of-late, AFPs have also been identified from freeze-tolerant plants. Plants arsenal against the freezing condition is quite diverse and composed of AFPs, antifreeze glycoproteins (AFGPs) and antifreeze glycolipids. AFGP of $S$. dulcamara requires the sugar moiety for its antifreeze activity as treatment of AFGPs with $\beta$-galactosidase reduces antifreeze activity (Duman, 1994). On the contrary, sugar moiety does not play pivotal role on the activity for AFGPs from other plants, since the de-glycosylated AFGPs exhibit similar antifreeze activity (Gupta and Deswal, 2012). Sequence analysis of AFGP from $S$. dulcamara reveals homology with the WRKY transcription factor. While other plant AFGPs are homologous to poly-galacturonase inhibitor protein (PGIP) (Gupta and Deswal, 2012). Plant AFPs on the other hand are mostly apo-plastic. However, AFPs from Prunus persica and Forsythia suspensa are intracellular, implicating their role in other signalling cascades and also in the inhibition of intracellular ice nucleation (Simpson et al., 2005). Sequence analysis of plant AFPs shows that they are homologous to the PR-proteins such as thaumatin-like proteins, class I and class II chitinases and $\beta$-1,3-glucanase (Hon et al., 1995). An interesting observation is the dual functioning of Plant AFPs. Plant AFPs exhibit both antifreeze and hydrolytic activities. Chitinases purified from cold-adapted winter rye and sea buckthorn show hydrolytic and antifreeze activities, likely $\beta-1,3$ glucanase also has been demonstrated to exhibit antifreeze activity and partial hydrolytic activities (14$35 \%$ ) at sub-zero temperatures. 
Structural information on plant AFPs are not much explored. Structure of Lolium perenne AFP is mostly explored. The protein is predicted to be a betaroll, with two flat surfaces composed of $\beta$-sheets on opposite sides of the protein. The predicted IBS from the mutational study is a flat $\beta$-sheet rich surface (Kuiper et al., 2001). CD spectroscopy study of several other plant AFPs reveals secondary structure of those proteins are mostly $\beta$-sheeted. However, AFP from A. mongolicus shows mixed topologies, where both $\beta$-sheeted and $\alpha$-helical residues are abundant in addition $\sim 55 \%$ of residues are in random coil conformations (Fei et al., 1994). 3-D structure predictions of 9 plant AFPs using homology modelling reveal most of them are $\beta$-sheeted, while glucanases from winter rye is mixed $\alpha$-helical and $\beta$-sheeted (Haas et al., 2013). Interestingly, asparagine residues are abundant in the IBS of plant AFPs. Lolium perenne AFP is consisted of 113 amino acids and there is sequence repeat of 7 semi-conserved amino acids (X-X-N-X-V-X-G). Mutational studies in carrot showed that upon mutation of asparagine with valine/ glutamine resulted significant loss of TH activity.

AFPs were also isolated from yeast Glaciozyma sp. AY30, known as LeIBP (Lee et al., 2010). It is also a right-handed $\beta$-helix which shows TH $\sim 0.42^{\circ} \mathrm{C}$. Several AFPs were also isolated from snow mold fungus (Typhula ishikariensis), known as TisAFPs (Xiao et al., 2009). Structurally, they are predicted to be highly similar with LeIBP and possess a hydrophobic ice binding surface.

First recombinant AFP from a marine diatom, C. Neogracile, was characterized in 2009 which showed TH activity $\sim 0.8^{\circ} \mathrm{C}$ (Kim et al., 2017).

Gilbert et al. first reported the antifreeze activity of 19 bacterial isolates during the screening of 866 bacterial colonies from an Antarctic lake (Gilbert et al., 2004). As time progressed, more and more AFPs have been isolated from different bacterial species from diverse habitat. However, the first solved crystal structure of a bacterial AFP is known as MpAFP, Marinomonas primoryensis (Garnham et al., 2008). The structure is a milestone in AFP research. It is a $\mathrm{Ca}^{+2}$-bound $\beta$-helical fold, where the perfectly oriented anchored clathrate water molecules were detected.

Structural diversity of the AFPs clearly indicates different evolutionary origin for different AFPs.
However, the selection pressure during evolution of this class of proteins remains same - i.e., to combat extremely cold temperature. In fact, it has been accepted that fish AFPs did not evolve at the time of evolution and speciation of Osteichthyes occurred $\sim 175$ million years ago (Jia and Davies, 2002). Evolution of AFPs is rather a very recent phenomenon. In Antarctic region, the glacial evolution pattern drastically changes $\sim 22$ million years ago (Cheng, 1998). During this period, the flow of warm sub-tropical water completely detached from the Antarctic circumpolar current flow. This event influences significantly modern Antarctic fish fauna, particularly the evolution of AFGP-bearing notothenoids. Comparison of gene sequences of ancestral and derived proteins reveals that the conversion of proteases to AFGPs occurred 5-14 million years ago. On the other hand, Arctic glaciation occurred much later $\sim 2.5$ million years ago. AFPs in northern fishes followed an independent evolution patterns. The selection pressure is not clear, rather is very complicated. Even sister species of sculpins bear completely different uncorrelated AFPs. Probably, different migration patterns and also freezing of their habitat occurred in different glacial period that make their need for the AFP evolution to occur independently.

\section{Measurement of Antifreeze Activity}

The activity of AFPs is commonly referred as ice recrystallization inhibition (IRI) activity and thermal hysteresis (TH) activity (Drori et al., 2014; Tomczak et al., 2003).

Ice crystals are unstable and constantly changes in number, shape and size. External perturbation strongly modulates the process, particularly the thermal fluctuations. During temperature fluctuation when the temperature increases, small ice crystal dissolves as a result unfrozen cold water amount increases. Now if there is mild depression of temperature, cold bulk water molecules add on the existing ice crystal to increase the size of the crystal, rather than start to nucleate a new ice crystal seed. This process is known as ice recrystallization (Olijve et al., 2016a). AFPs are known ice recrystallization inhibitors however the exact mechanism by which they exhibit the activity is unknown. AFGPs are particularly potent ice recrystallization inhibitors (IRI) (Congdon et al., 2013) 
and hyperactive insect AFPs which show higher TH activity, exhibit weak IRI activity (Kim et al., 2017). Clearly there is no obvious correlation between $\mathrm{TH}$ and IRI activity. IRI active AFPs have strong application potential in cryopreservation, because during freeze-thaw cycle, recrystallization is a common phenomenon.

Fish AFPs were first experimentally tested for their ability to inhibit ice recrystallization, using "splat cooling" process (Knight et al., 1988). In this method, a small water droplet of AFP solution has been dropped to a metal disc which is highly cooled with the aid of liquid nitrogen. As soon as the sample hits the disc, it becomes frozen and the frozen ice layer is then placed under microscope at high sub-zero temperature such that the sample starts to recrystallize over time. However, a known problem in the process is endpoint of IRI activity, where the activity loss happens beyond a certain concentration limit. Later, the group of P. L. Davies developed a method for IRI activity measurement of AFP solutions, using a capillary based method that allows direct visualization of the samples under microscope in the same field (Tomczak et al., 2003). Briefly, samples are loaded using $10 \mu$ glass capillaries and align in such a way that there is no space between adjacent capillaries. The total capillary series are then rapidly frozen at $\sim-50^{\circ} \mathrm{C}$ in anhydrous 2,2,4-trimethylpentane. After complete freezing, samples are dipped in the same solution at $-6^{\circ} \mathrm{C}$ and incubated overnight. Images are taken at the start and endpoint of the incubation, using high intensity illuminator in a dry air chamber.

Type III AFPs and AFGPs are potent ice recrystallization inhibitors (Congdon et al., 2013; Congdon et al., 2016; Tomczak et al., 2003). Carpenter et al., demonstrated that winter flounder AFP provided cryo-protection to red blood cells in hydroxyethyl starch solutions (Carpenter and Hansen, 1992). However, they also noticed that high AFP concentrations $(1.54 \mathrm{mg} / \mathrm{ml})$ induce cell damage, although effectively inhibited extracellular recrystallization. A fungal AFP isolated from psychrophilic yeast Glaciozyma sp. AY30, LeIBP, is moderately active but shows better protection to mouse ovarian tissues than hyperactive AFPs due to its high IRI activity (Lee et al., 2015). Type I AFP, on the other hand, has been shown to provide cryopreservation of Sparus aurata embryos during different developmental stage (Robles et al., 2007). Type III AFPs were used to provide cryopreservation to mature mouse oocytes (Jo et al., 2012), chimpanzee spermatozoa (Younis Abdelmoneem et al., 2013) and mature bovine oocytes (Chaves et al., 2016). Even, type III AFPs were used successfully in the cryopreservation of transplanted mammalian heart (Amir et al., 2003). However, dynamic ice shaping (DIS) i.e., shape alteration of the growing ice crystal due to the specific ice plane binding, is a common problem associated with IRI. Also recently, it has been demonstrated that high AFP concentrations induce toxicity in cryoprotected human embryonic liver and human embryonic kidney cells (Liu et al., 2007a). These facts recently stimulate research on designing new AFP-mimetic with improved IRI activity without DIS and with reduced toxicity even in high concentration (Congdon et al., 2013; Laezza et al., 2017; MacDonald et al., 2017; Olijve et al., 2016a).

Thermal hysteresis (TH), on the other hand, is non-equilibrium depression of hysteresis freezing point below the melting point (Kristiansen and Zachariassen, 2005). AFPs demonstrate the TH activity by creating a thermal hysteresis gap between hysteresis freezing and equilibrium freezing point. AFPs lower freezing point in a non-colligative manner. Degree of $\mathrm{TH}$ depends strongly on the type of AFP (Kim et al., 2017). AFGPs exhibit TH activity within the range of 0.1 to $0.6^{\circ} \mathrm{C}$ in the concentration range of $10-40 \mathrm{mg} / \mathrm{ml}$ (Wilkinson Brendan et al., 2012). The TH activity of type I AFPs are almost similar with AFGPs, but within a lower concentration range $2-12 \mathrm{mg} / \mathrm{ml}$ (Kun and Mastai, 2007). On the other hand, Type III AFPs show TH activity of $\sim 1-1.5^{\circ} \mathrm{C}$ at a concentration of $20 \mathrm{mg} / \mathrm{ml}$ (Pertaya et al., 2008). Type II AFPs show thermal hysteresis up to $0.7^{\circ} \mathrm{C}$ at a concentration of $10 \mathrm{mg} / \mathrm{ml}$ (Gronwald et al., 1998). Reports on the activity of type IV AFP are not much available. Gauthier et al., reported TH activity of $0.125^{\circ} \mathrm{C}$ at a concentration $\sim 2.5 \mathrm{mg} / \mathrm{ml}$ for a type IV AFP (Gauthier et al., 2008). Insect AFPs are much more active than the fish AFPs, termed as hyperactive. Insect AFPs show hysteresis of $4-6^{\circ} \mathrm{C}$, even $0.06-0.07 \mathrm{mg} / \mathrm{ml}$ of protein can give $0.6-0.7^{\circ} \mathrm{C}$ thermal hysteresis (Hakim et al., 2013). Later, hyperactive AFPs also have been identified from fish, arthropod and in Antarctic bacterium.

The age-old technique for the determination of TH activity is nanoliter cryoscopy (Knight et al., 1984; 
Olijve et al., 2016b). In this process, experiments are carried out under a microscope. Initially a nanoliter aqueous droplet is flash-frozen and then slowly has been melted to obtain a single ice crystal. After that, the sample is allowed to cool very slowly up to the point where burst growth happens. TH activity is the difference between the melting point and the point where burst growth initiates. The primary advantage of this technique is that it requires very little AFP solution (in nanolitre). However, the obtained activity is highly process-dependent and many factors strongly influence the observed activity, particularly, the rate at which melting and cooling occurs, initial size of ice crystal nuclei. Takamichi et al., evaluated different experimental conditions on the observed $\mathrm{TH}$ activity of a type III AFP from eelpout (Takamichi et al., 2007). It was demonstrated that fast cooling process dramatically decreases the TH activity. Annealing time has a profound influence on the TH activity and in type III AFP, 2-3 hours annealing time increased the measured activity. The observed effect has been rationalized in terms of adsorption-inhibition model. Authors suggested that AFP during the annealing period binds to additional ice layer through its convex surface which enhances the antifreeze activity.

Sonocrystallization was much later developed to overcome the effect of experimental condition on the measured TH activity (Olijve et al., 2016b). It is also highly accurate. Here, the sample is first slowly super cooled up to $\sim-6^{\circ} \mathrm{C}$. Then a short ultrasound pulse is given to initiate nucleation and freezing. Once the freezing starts, the latent heat is released which increases the sample temperature. The latent heat is then transferred to increase the surrounding heat bath from $-6^{\circ} \mathrm{C}$ to $+0.4{ }^{\circ} \mathrm{C}$. However, the sample temperature response is very slow towards this temperature fluctuation and remains almost constant during the heating process. In presence of AFPs in solution, the ice growth is inhibited which resulted the sample temperature quickly approaches to the bath temperature. Therefore, the increment saturates at the equilibrium melting temperature of the solution. $\mathrm{TH}$ is then calculated as a difference between the melting and freezing temperatures.

Insect AFPs are hyperactive when their activities have been determined using nanoliter cryoscopy, however, they are found to be moderately active during sonocrystallization measurement. On the other hand, activity of type III AFPs has been found to be few orders of magnitude lower than the insect AFPs, when determined using cryoscopy but in sonocrystalization they are often reported to be more active than insect AFPs. The observed differences in the TH activity are later explained in terms of binding plane specificities (Olijve et al., 2016b). Since antifreeze activity exhibited by AFPs is kinetically driven and ice growth rate on different ice planes are dramatically different. Ice grows with much faster rate along the a-axis (primary prism plane) as compared to the c-axis (basal plane). In cryoscopy, the cooling rate is slow, therefore both prism and basal plane binding is critical. Insect AFPs bind to both, the basal and prism planes, and therefore exhibit highest degree of activity in cryoscopy. However, in sonocrystalization, the rate of cooling is very fast. Therefore, slow growing basal plane binders show reduced activity in sonocrystalization. Type III AFPs preferentially bind to the prism plane and stop growth on the fast growing prism plane. Therefore, type III AFPs show comparatively higher TH activity in sonocrystallization.

\section{AFPs are Specific Binders Towards Different Ice Planes}

According to the "adsorption inhibition model", AFPs adhere on the growing ice surface. However, characterization of such ice-protein interaction is very difficult due to the dynamic character of the ice/water interface (Hayward and Haymet, 2001; Karim and Haymet, 1988). One of the early techniques used to identify ice plane binding specificity is ice-etching technique (Knight et al., 1991). In this process, initially deionized and filtered water is allowed to freeze slowly in an insulated glass container at $-1^{\circ} \mathrm{C}$. After two days, there is formation of a single large ice crystal at the top surface with its $\mathrm{C}$-axis oriented vertically. Orientation of the a-axis has been determined by allowing growth of a little frost on the ice surface and observing the location of the prism faces, which are parallel to the a-axes. Then, a $2 \mathrm{~cm}$ width ice slab is cropped from the ice using a band saw in such a way that the cut surfaces are parallel to the prism plane. These sections are stored in such a way that the cut surfaces are in contact. This allows generation of prism face. This section is then cut into several cubes of $2 \mathrm{~cm}$ dimension and used as a seed for growth experiments. 


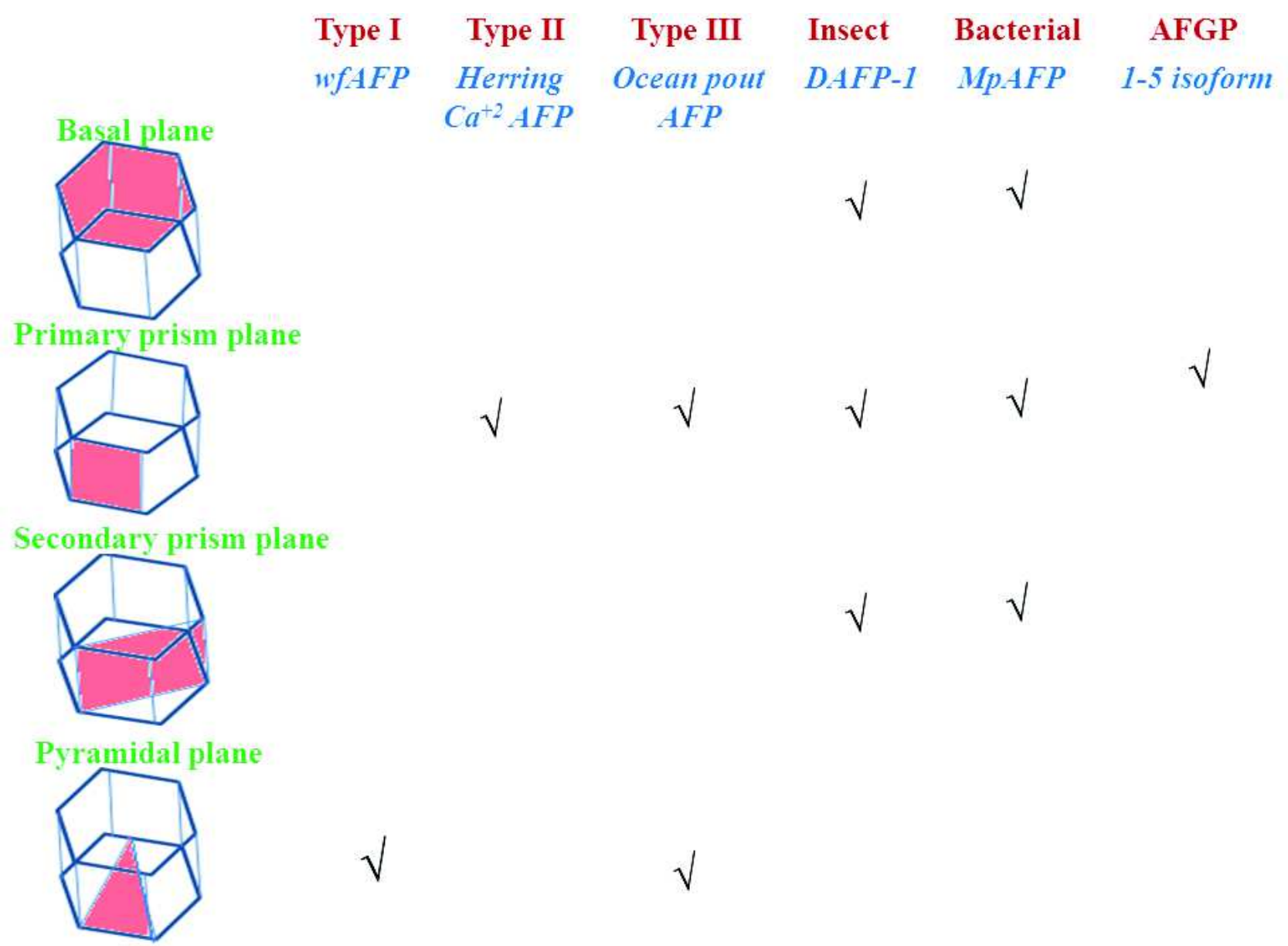

Fig. 2: Binding plane specificity summary of different classes of AFPs

The ice seed is then attached to a brass cold finger in such a way that the prism face is normal to the long axis of the finger. The cold-finger-ice cube is then inserted into a dilute AFP solution taken in an insulated cylindrical container at $15^{\circ} \mathrm{C}$. Initially, the seed is melted and then is grown for 6 hours into a single crystal hemisphere. The hemisphere is then removed and transferred to a cold room at $\sim-10^{\circ} \mathrm{C}$. Then the surface of the hemisphere is scrapped with a blade to eliminate frozen AFP solution and allowed to evaporate in the cold room. During the process, the surface smoothens, except the region where AFPs are incorporated, resulting an etching pattern. These etch patterns help to identify the AFP binding planes.

Several etching experiments of different AFPs reveal that AFPs are specific ice plane binders (Fig. 2). Type I AFPs specifically bind to the pyramidal plane (Knight et al., 1991). Insect AFP from Choristoneura fumiferana specifically binds to both prism and basal plane, evident from etching experiments (Graether et al., 2000). However, the binding plane specificity of type III AFPs is controversial. Initial etching experiments suggest that type III AFP interacts with the prism plane. Antson et al. later refined the etching data and reported that type III AFPs bind to different ice planes that are parallel or inclined at a small angle to the c-axis, since the surface coverage in the etching experiment shows a broad coverage feature. Later, it has been proposed that type III AFPs can bind to both primary prism and pyramidal plane with favourable affinity (Antson et al., 2001). Ice-etching experiments suggest that type II AFPs bind to the primary/secondary prism plane with considerable affinity (Cheng and DeVries, 1991; Liu et al., 2007b).

Recently, the ice-etching technique has been replaced by fluorescence-based ice plane affinity (FIPA) analysis (Basu et al., 2014). In the FIPA 
experiments, the protein is tagged with a fluorescence marker or often covalently attached to a dye. Then, the labelled protein is slowly incorporated into a large single ice crystal, which is in the shape of a hemisphere. The properly aligned AFP-incorporated ice hemisphere is then imaged under UV light to observe the fluorescence of the bound AFP on a particular plane. During the imaging, non-specific signals are blocked by using filters. Notably, it has been observed that the effects of labelling are minimal on the adsorption of protein to a particular ice plane. This technique allows direct visualization of an AFP to a specific ice plane; even it preferentially binds to multiple planes. FIPA analysis complements well with the traditional ice-etching data. It has been demonstrated in FIPA experiments that hyperactive insect AFPs are basal plane binders, whereas moderately active AFPs are essentially non-basal binders.

\section{AFP Binding to an Ice Surface: More Surprises}

Nature of AFP binding to the ice surface is another controversial topic. It was assumed to be irreversible in nature. Because, if the process is reversible when the bound AFP detaches from the growing ice surface, there is further growth of the ice front. Thus, ice growth cannot be stopped completely. However, this irreversible binding mode was later challenged critically. Particularly, ice-water interface is not a rigid surface that can provide platform for strong binding; rather it is a dynamic layer, where the interface smoothly converges from the solid ice layer to the bulk through several layers of water, where the order reduces gradually. In addition, later it has been demonstrated that AFPs show concentration dependent antifreeze activity which indicates a dynamic equilibrium between bound and in solution AFP. In support to this hypothesis, Q.Z. Li developed a 2-D kinetic model for AFP adsorption on ice surface and reproduces the concentration-dependent $\mathrm{TH}$ curves for two insect AFPs from Tenebrio molitor and Choristoneura fumiferana (Li et al., 2006). According to the model, antifreeze activity depends on the surface coverage of the AFP on ice. There is a delicate balance between the size of AFP and the TH activity. Initially, up to a certain size limit, with increasing size the surface coverage increases which leads to an enhancement of the activity. After a critical limit increasing size of the protein increases the flexibility of the protein, which results in a reduction of AFP adsorption to the ice surface. Also, they have shown that the activity depends on the area of surface coverage by the AFP not only the strength of the AFPice interactions. This mode of binding clearly supports reversible AFP-ice binding. Recently, with the aid of microfluidics and temperature controlled systems, the role of bound and solution AFPs on the concentration dependent $\mathrm{TH}$ activity has been critically explored. Celik et al., recently showed that GFP-tagged insect AFP strongly adheres on the ice surface (Celik et al., 2013). The interactions appears to be up to the limit of irreversibility since gradual removal of AFP greatly reduces the solution AFP concentration but hardly reduces bound AFP pool. Recently, direct binding of AFP to the ice surface mediated through a layer of water has also been proposed (Chakraborty and Jana, 2017a; Chakraborty and Jana, 2017b; Chakraborty and Jana, 2018).

Another interesting observation is the timedependent TH activity of the AFP. It has been reported that the activity of type I AFP enhances five times during exposure to ice crystal for $180 \mathrm{~min}$. Increase in activity with increasing the annealing time is also reported for type III AFP. Recently, Drori et al., used fluorescence microscopy in connection with microfluidics to explore critically time-dependence of the TH activity of type III and hyperactive AFP (Drori et al., 2014). Interestingly, they observed that the activity of hyperactive AFP is highly time-dependent and increases as much as 40 times with changing the incubation time from few seconds to hours. However, moderately active AFPs do not demonstrate such strong time dependent activity. This differential timedependent activity can be rationalized in terms of their binding plane specificities. Hyperactive AFPs are slow progressive basal plane binders and their adsorption kinetics is multi-exponential in nature. Takamichi et $a l$, indicates the possibility of secondary binding in the process of adsorption (Takamichi et al., 2007) also. However, type III AFP does not show such timedependent TH activity. This group of AFP binds to the fast growing prism plane rather than the slow growing basal plane.

\section{Antifreeze Activity of AFP: A Macroscopic View}

Raymond and DeVries first proposed a model that provides macroscopic view on the working mechanism of antifreeze activity of the AFPs, known as 
"adsorption-inhibition model" (Raymond and DeVries, 1977). According to this model, AFPs bind to the ice surface irreversibly. Due to the presence of bound AFP on the ice surface, ice growth is only possible on the exposed ice surface in between two bound AFPs. As a result of this limited access to the existing ice seed, the newly grown ice front becomes curved. Thus in presence of several bound AFPs; the ice front is grown as several small convex surfaces. As more and more water is added to the curved ice front, the curvature of the newly grown ice surface increases and ultimately the ice growth halts at a critical limit. The process is summarized in Fig. 3.

\section{Adsorption-Inhibition model}
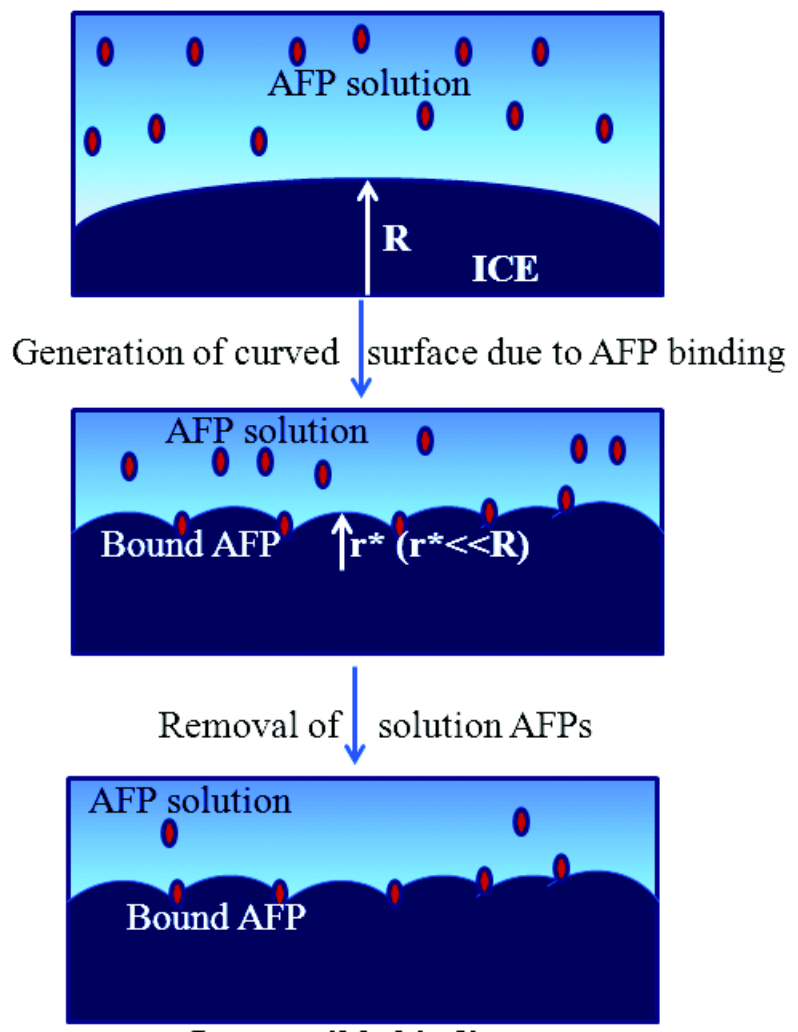

Irreversible binding

Fig. 3: Pictorial representation of the "AdsorptionInhibition model"

Thermodynamically, the process can be explained in terms of "Kelvin effect". At the melting temperature, the water and the ice phase are in equilibrium which implicates that the free energy of both the phases are equal. Below the melting temperature, free energy of the ice phase becomes lower; as a result the free energy difference between two phases increases. The difference is more with further decrease in the temperature. The phenomenon can be analytically explained by eq. 1

$$
\Delta \mathrm{G}=(\Delta \mathrm{H} . \Delta \mathrm{T}) / \mathrm{T}_{\mathrm{m}}
$$

$\Delta \mathrm{G}$ is the free energy difference per unit volume, $\Delta H$ is the heat of fusion and $\Delta \mathrm{T}$ is the degree of super cooling $(K) . T_{m}$ is the melting temperature $(K)$.

The effect of curved surfaces on the energy equilibrium can be explained in terms of the pressure effects that exist across the curved surfaces. The pressure difference $(\mathrm{P})$ across an interfacial curved surface can be represented by eq. 2

$$
\Delta \mathrm{P}=2 \sigma / \mathrm{r}
$$

$\sigma$ is interfacial tension and $r$ is the radius of the curved surface. For a convex surface, as the surface curvature increases, the interfacial pressure increases. As a result free energy of the solid phase increases continuously with increasing the curvature of the convex surface, since for a non-compressible system like ice

$$
\Delta \mathrm{G}=\mathrm{V} \Delta \mathrm{P}
$$

Thus after certain curvature limit the free energy differences between the solid and liquid phase diminishes which stopped the growth of the convex surface. The critical curvature limit after which the ice growth stopped also can be determined.

$$
\begin{aligned}
& \Delta \mathrm{G}_{\mathrm{v}}=\Delta \mathrm{P} \\
& (\Delta \mathrm{H} \cdot \Delta \mathrm{T}) / \mathrm{T}_{\mathrm{m}}=2 \sigma / \mathrm{r} \\
& \mathrm{r}^{*}=2 \sigma \mathrm{T}_{\mathrm{m}} /(\Delta \mathrm{H} \cdot \Delta \mathrm{T})
\end{aligned}
$$

The model is a well-accepted model for ice growth inhibition mechanism by AFP. However, there are some facts that are not in accordance with the model. The model explained thermal hysteresis in terms of the curved growth surfaces in presence of bound AFPs. If that is true then it is expected that during the process of melting, the interface becomes concave in presence of bound AFP which ultimately leads to superheating. But in reality, it has been observed that ice melts at the melting temperature in presence of bound AFPs. Also, according to this model surface density of the AFPs is the most crucial determinant. Thus different AFPs in same concentration are expected to be similarly active. However, the antifreeze activity is strongly dependent 
on the class of the AFPs where they belong. Insect AFPs are hyperactive. Evident from their crystal structure, the topology of this class of AFP is completely different than the other classes. Topology as well as nature of the ice binding site has strong influence on the activity of AFPs. Thus the macroscopic picture is a qualitative description of the observed TH activity of the protein, one need to go to the atomistic level to provide mechanistic insight into the process quantitatively.

\section{Mechanism of Ice Recognition by AFPs: A Structural Insight}

Atomistic insight into the process of ice binding remains elusive and highly controversial. Very early thought regarding the ice recognition by AFP was hydrogen bond matching hypothesis originally proposed by Lin and DeVries (Devries and Lin, 1977). They have analysed sequence of $\alpha$-helical type I AFP from winter flounder and noted that there exists periodicity in the location of polar residues. Distances between two polar residues THR and ASP is $4.5 \AA$ and there are three such pair protrudes from one side of the helix and the side chains of hydrophobic residues are oriented away from this face, according to their spacefilled model. Very interestingly, the distances match exactly $(4.51 \AA)$ with the oxygen atom repeat distances along the a-axis. Polar groups have side chains capable of hydrogen bond formation with the ice surface oxygen. Thus they proposed that hydrogenbonding between ice and AFP is the primary binding force. Later in ice etching experiment, it was identified that the AFP preferentially binds at the pyramidal plane however; the authors still defended the hydrogen bond matching hypothesis. They can model the AFP on the pyramidal plane where they observed periodicity between two another polar groups which matches to the $16.5 \AA$ repeat distance of the pyramidal plane (Wen and Laursen, 1992b). This observation motivated many computational studies to identify the binding orientation of the type I AFP on pyramidal plane (Madura et al., 1994; Wen and Laursen, 1992a). All of them are stochastic conformational search process to find optimal hydrogen bonds between AFP and ice. It was concluded that the preferred biding orientation of the AFP on $<2021>$ pyramidal plane is in $<0112>$ direction where four equal-spaced THRs forms hydrogen bonds with the pyramidal plane. However, initial modelling studies do not include the effect of solvation and also the ice-water interfacial heterogeneity is completely ignored in their calculations. Later in 1997, Cheng et al., although included the effect of solvation during the simulation of the AFP on ice surface, their trajectories were very short to analyse the complete adsorption picture, also their motivation was to find the hydrogen bond matching between the IBS of AFP and ice plane (Cheng and Merz, 1997). However, still they noticed that hydrophobic hydration contributes significantly to the binding affinity during the adsorption of the AFP on ice surface. In 1996, Jia et al., identified and solved crystal structure of type III AFP from eel pout (Macrozoarces americanus) and observed that the flat amphipathic surface forms five hydrogen bonds with the $<1010>$ prism plane in $<0001>$ direction (Jia et al., 1996). However, in this binding mode, authors fail to provide explanation on the loss of activity of the ASN14SER mutant ( $75 \%$ loss of activity), because side-chain of ASN14 oriented away from the ICE plane, thereby cannot form hydrogen bonds with the prism plane. Author justifies the mutation data by proposing a complex binding mechanism for type III AFP where it can bind to multiple surfaces of the growing ice plane. Later in 2001, Antson et al., proposed a different binding model for type III AFP. Their ice-etching experiments showed that indeed the binding surface of the AFP is complex and suggest a preferential binding to the pyramidal plane guided by hydrogen bond matching and van der Waals interactions driven tight packing of apolar residues on ice surface (Antson et al., 2001). It is noteworthy that their modelling study did not consider the explicit ice/water interface. The hydrogen-bond matching model was rejected when mutational data on type I AFP were published (Zhang and Laursen, 1998). Winter flounder AFP contains four threonine residues at $2^{\text {nd }}, 13^{\text {th }}, 24^{\text {th }}$ and $35^{\text {th }}$ position. Mutations of THR residues with SERs completely abolish the antifreeze activity, although SER has side chain capable of hydrogen bond formation like THR. However, upon mutation of THRs with VALs (non-polar side chain), the AFP still remain $\sim 30 \%$ active. However, none of these mutations appear to alter the structure of the AFP, evident from circular dichroism study. This observation nullified the hydrogen bond matching hypothesis and finding an alternative binding model became a topic of interest. Crystal structures of many AFPs have been solved later, these includes, type III 
AFPs, hyperactive insects AFPs. Analysing all these structures of AFPs reveal an interesting feature. In general, AFPs possess a flat, rigid and hydrophobic IBS, exceptions are type I AFPs and AFGPs. These observations give rise to a new model, the "snug-fit model" (Baardsnes and Davies, 2002; DeLuca et al., 1998; Yang et al., 1998). According to this model, the flat surface of AFP fits appropriately on an ice plane guided by surface complementarity driven tight packing. According to this model hydrogen bonding interactions between the ice binding plane (IBS) of the AFP and the ice surface can complement but not the necessary criterion for ice binding. The model also finds credential from several mutational data from type III AFP where it has been shown that substitution of residues at the IBS with amino acids bearing bulkier side chain is detrimental to the antifreeze activity of the AFP (DeLuca et al., 1998). However, the model suffers criticism from several computational studies. Now it is well accepted that ice/water interface has a diffusive structure and such diffuse region is not that rigid to provide surface complementarity to the IBS of AFP. Such interactions are not sufficient enough to give rise irreversible binding of AFP to ice surface, which is a prerequisite according to the adsorptioninhibition model.

Recently, AFPs came into intense research focus for their unusual hydration pattern. Nutt and smith performed molecular dynamics simulations of a hyperactive insect AFP (Choristoneura fumiferana) at $300 \mathrm{~K}$ and $250 \mathrm{~K}$ (Nutt and Smith, 2008). They observed that water dynamics is more retarded around the IBS in comparison to the nonIBS planes. They implicated the presence of ordered water around the IBS is a prerequisite to bind AFP to the ice surface. However, they did not consider the ice slab in their simulations. Ebbinghaus et al., using terahertz spectroscopy showed that AFGPs can induce long range perturbation on water dynamics and the author argued that the direct modulations of water by the AFGPs prevent it from freezing (Ebbinghaus et al., 2010). Thus, direct binding is not needed to exhibit antifreeze activity. Previously, Wierzbicki et al. showed that winter flounder AFP adsorbed at the ice/water interface rather than its binding to ice plane (Wierzbicki et al., 2007). Later, many simulations demonstrated that presence of AFP at ice/water interface alter local ice growth kinetics even induces ice melting (Todde et al., 2015; Todde et al., 2014). Recent microfluidic experiments clearly showed that AFP indeed adhere on the ice surface and the binding is strong enough up to a point of irreversibility (Celik et al., 2013). Later Meister et $a l$., showed that both short-range interaction and longrange protein induce water dynamics plays major role in dictating antifreeze activity of hyperactive insect AFPs (Meister et al., 2013). The same group in 2014 reported the presence of highly ordered ice-like water around the IBS of a type III AFP at lower than ambient temperature (Meister et al., 2014). Interestingly, mutation of an IBS residue, THR18ASN, completely abolishes the ordered water populations. All these observations and several simulation studies (Chakraborty and Jana, 2017a; Chakraborty and Jana, 2017b; Chakraborty and Jana, 2018; Midya and Bandyopadhyay, 2014; Midya and Bandyopadhyay, 2017; Nutt and Smith, 2008) suggest that, the hydration around the IBS plays crucial role on ice recognition by AFPs, however, the details of the mechanism are completely lacking since probing hydration water around a protein surface in presence of ice/water interface is difficult with experiments and also computationally challenging.

\section{Clathrate Mediated Ice Recognition by AFP: Emergence of a New Paradigm}

Winter flounder AFP, is a 37 residue helical type I AFP where it was demonstrated that THR->SER mutation leads to complete loss of activity, however VAL mutation retained $\sim 30 \%$ activity, although the authors argued that the substitution does not alter the structure of the protein by doing circular dicroism spectroscopy (Zhang and Laursen, 1998). Circular dicroism spectroscopy is widely used to elucidate the secondary structure of a protein but does not talk about the dynamics of the protein. We have performed equilibrium simulation and metadynamics simulation to explore the effect of mutation on the structure and dynamics of the AFP (Chakraborty and Jana, 2017a). Results are summarized in Fig. 4. The thermodynamically stable minimum of the wild-type AFP is a straight helix. However, the free-energy minima of the SER-mutant is helical but is not a straight helix, rather it is in kinked form. Clearly, in the kinked form, the IBS is disrupted and therefore unable to bind to the ice surface. In this form helicity remains unaltered compared to the wild-type, which explains why circular dicroism spectroscopy detects no 


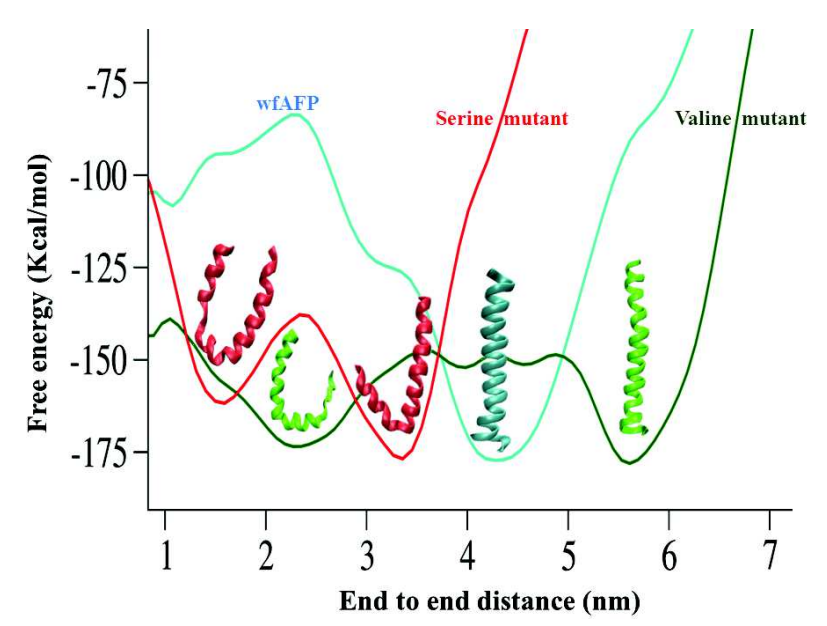

Fig. 4: Free-energy profiles obtained from metadynamics of the $w f A F P$ (cyan) and its valine (green) and serine (red) mutants are shown. Lowest energy structures are shown in ribbon representation and colour according to their free energy profile

deviation in comparison to the wild-type. Free energy simulation reveals two populations for VAL-mutant, a straight helix and a bent conformation. The bent conformation of VAL mutant is unable to bind at the ice surface due to its disrupted structure; however the straight conformation retains helical form like the wild-type.

Further potential of mean force calculations have been carried out to identify the bound conformation of the AFP on ice surface, particularly for the VAL mutant, where the hydrogen bonding side chains are missing. Notably, all the simulations have been carried out in realistic systems, where an ice slab is present within a water box and the width of the ice/water interface is $\sim 13-15 \AA$, after that there is bulk water. Interestingly, the PMF minima correspond to a state, where the wild-type and VAL mutant adsorbed on the pyramidal surface, although the simulation started from hydrogen bonded state, where AFP forms hydrogen bonds with the ice surface. In this adsorbed state the methyl group faces towards the ice surface and there is formation of clathrate-like structure around the methyl group involving surface of the ice slab (Fig. 5). In low temperature regime, we found clear evidence of formation of such water structure around the methyl groups at the IBS for both HPLC6 and its VAL mutant. We propose that the ordered hydration water helps AFP to first adhere on the ice surface, then

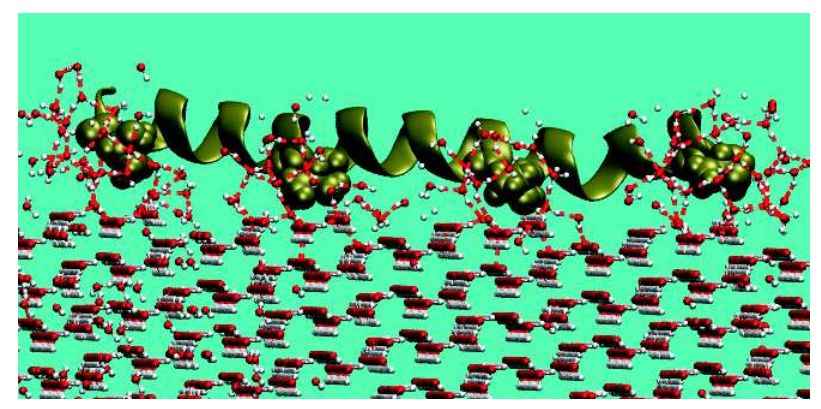

Fig. 5: Adsorbed structure of the wfAFP to the pyramidal plane is shown. AFP is shown as green ribbon where four THRs are represented in VdW mode. Ice surface and interfacial water that forms water clathrate around the THRs are shown in CPK mode. Hydrogen bonding in the water clathrate structures is represented as red dotted line

release of some hydration water and involvement of ice surface water to complete the caging around the methyl group in the bound state. Presence of ordered water shell around the hydrophobe and its size, shape and temperature dependency has been thoroughly studied, recently (Halder and Jana, 2018; Parui and Jana, 2017; Samanta and Bagchi, 2018; Samanta et al., 2018).

The clathrate mediated ice recognition was then further tested for a hyperactive insect AFP, Spruce budworm, $s b w$ AFP isoform 501 (Chakraborty and Jana, 2017b). Equilibrium simulation $\sim \mathrm{T}_{\mathrm{m}}$, reveals that the perfectly aligned threonine residues at the IBS induce water ordering due to the combined effect of hydrophobic hydration and confinements. Hydroxyl groups of the threonine residues further stabilize the water network which results in the "anchored clathrate water". These water molecules are crystallographically resolved in the crystal structure of $s b w$ AFP and due to their excellent match with the ice surface, these water molecules have been predicted to play crucial role in ice recognition (Garnham et al., 2011). Interestingly, similar clathrate mediated ice recognition mechanism has been observed for insect AFP. The adsorbed state corresponds to a state where AFP is accumulated on the ice surface through a network of highly ordered water around the IBS. Perfectly aligned methyl side chains of THRs residues at the IBS facilitate water clathrate formation. Ice surface and anchored clathrate water molecules are also involved to form water clathrate around the THR ladder. Anchored clathrate water makes co-operative dual 
hydrogen bonds involving both IBS of AFP and the ice surface thus effectively anchor AFP on the ice plane. It appears that the clathrate mediated ice recognition is a unanimous mechanism applicable to different classes of AFPs.

This binding model was also used to shed light on the pronounced time-dependent $\mathrm{TH}$ activity of insect AFP adsorbed on basal plane (Chakraborty and Jana, 2017b). We have modelled the process by performing simulations at two different temperatures $(225 \mathrm{~K}$ and $210 \mathrm{~K})$. We have used SPC/E water model, which has a melting point of $215 \mathrm{~K}$. So, the simulation at $225 \mathrm{~K}$ has been used to find the nature of initial adsorption during the point of initial contact at ice/water interface, where there is no growth of ice front. Free energy and equilibrium simulations reveal that insect AFP adsorbed on basal plane with the aid of a layer of water. This layer of water effectively anchors the AFP on ice plane, thus forming a stable adsorption complex. Further characterization reveals that this layer of water is clathrate-like in nature. However, during simulation of this adsorbed complex at lower temperature at $210 \mathrm{~K}$ (ice growth simulation) reveals an interesting feature. The intermediate clathrate water which anchors AFP on basal surface becomes ice-like. Thus, with further growth of one layer of ice front, this anchoring water becomes part of the growing ice-front and AFP directly binds to the growing ice front mediated by hydrogen bonds, thus permanently attaches to the ice slab. This clathrate-like to ice-like transition of the water layer that anchors the AFP on ice surface is the origin of time-dependent $\mathrm{TH}$ activity. Also, the dynamic transition from an adsorbed state to a permanently bound state has been predicted to be the origin of nearly irreversible binding as observed from recent fluorescence-tagging based microfluidic experiments. The mechanism of ice recognition of insect AFP and the origin of time-dependent TH activity is graphically represented in Fig. 6 .

We have further tested the mechanism to explain the binding plane-specificity data for hyperactive insect AFP. Potential of mean force calculations reveal the order of binding affinity is: basal plane $>$ prism plane $>$ pyramidal plane matches well with the ice-etching data. The observed binding affinity is strongly correlated with the number and their instantaneous fluctuations of the anchored clathrate water (Chakraborty and Jana, 2018). On basal plane, there are optimal numbers of anchored clathrate water molecules that are highly stable leading to highest binding affinity. On the prism plane, although there are equivalent numbers of anchored clathrate water in between the IBS and ice surface but their fluctuations are high which reduces the binding affinity for AFP adsorption. In the pyramidal plane, number of such water molecules is less and also their fluctuations are high, leading to the least affinity of $s b w$ AFP towards the pyramidal plane.

\section{Future Directions}

The proposed hydration water mediated ice adsorption

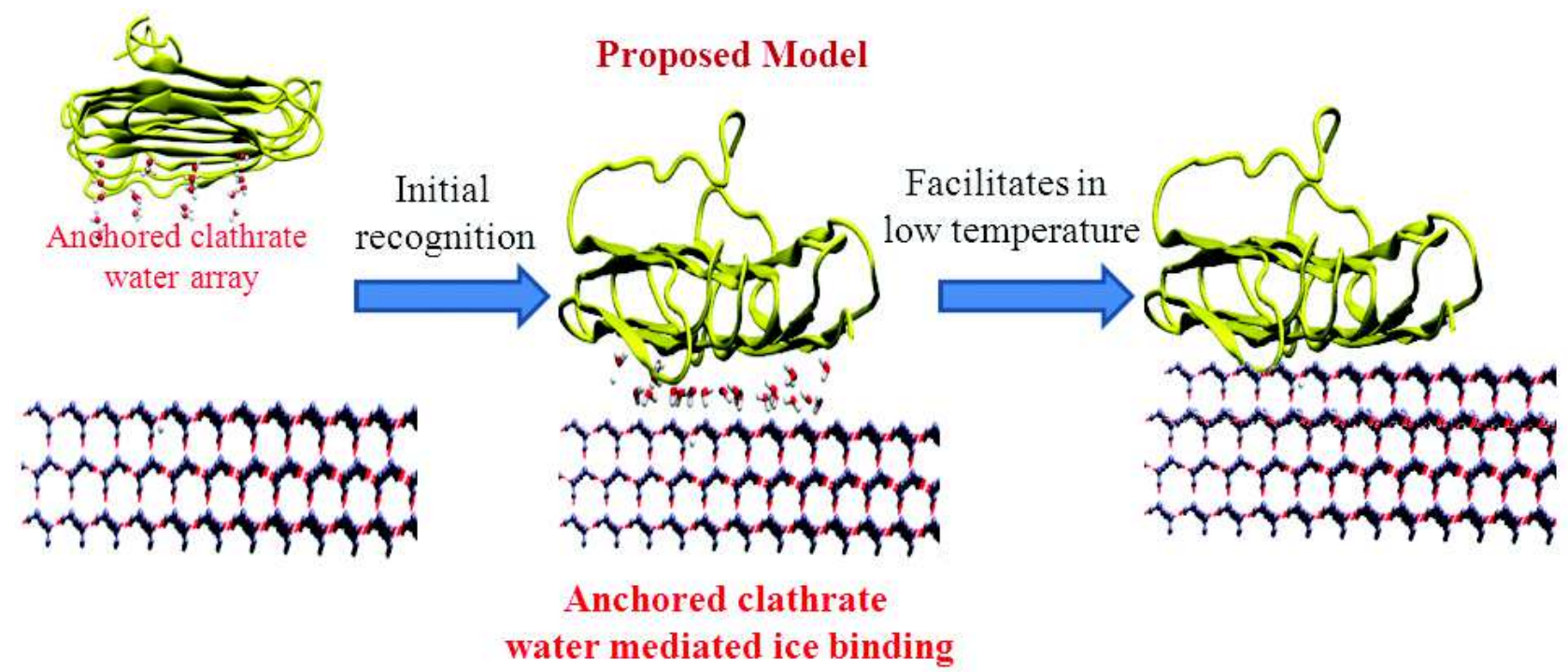

Fig. 6: The mechanism of ice recognition of insect AFP and the origin of time-dependent TH activity is shown 
hypothesis appears to be a promising model, applicable to different types of AFPs and quantitatively explains mutational data, experimentally observed binding plane specificity data. Moreover, the model directly links the unusual hydration of AFP to the ice recognition. However, generality of the model has to be tested further with other classes of AFPs. Also the hypothesis needs to be verified experimentally. However, characterizing ice/water interfacial water and detecting water orientations is highly challenging for experimentalists. Recent developments in heterodyne detected sum frequency generation spectroscopy generate a ray of hope in characterizing the bound state of AFP on ice surface. The technique is highly surface-specific and has been used to probe ice/water

\section{References}

Amir G, Rubinsky B, Kassif Y, Horowitz L, Smolinsky A K and Lavee J (2003) Preservation of myocyte structure and mitochondrial integrity in subzero cryopreservation of mammalian hearts for transplantation using antifreeze proteins - an electron microscopy study European Journal of Cardio-Thoracic Surgery 24 292-297

Antson AA, Smith D J, Roper D I, Lewis S, Caves L S D, Verma C S, Buckley S L, Lillford P J and Hubbard R E (2001) Understanding the mechanism of ice binding by type III antifreeze proteins 11 Edited by K Nagai Journal of Molecular Biology 305 875-889

Baardsnes J and Davies P L (2001) Sialic acid synthase: the origin of fish type III antifreeze protein? Trends in Biochemical Sciences 26 468-469

Baardsnes J and Davies P L (2002) Contribution of hydrophobic residues to ice binding by fish type III antifreeze protein Biochimica et Biophysica Acta (BBA) - Proteins and Proteomics 1601 49-54

Basu K, Garnham C P, Nishimiya Y, Tsuda S, Braslavsky I and Davies P L (2014) Determining the Ice-binding Planes of Antifreeze Proteins by Fluorescence-based Ice Plane Affinity JoVE e51185

Buch J L and Ramløv H (2017) Detecting seasonal variation of antifreeze protein distribution in Rhagium mordax using immunofluorescence and high resolution microscopy Cryobiology 74 132-140

Carpenter J F and Hansen T N (1992) Antifreeze protein modulates cell survival during cryopreservation: mediation through influence on ice crystal growth Proceedings of the National Academy of Sciences $\mathbf{8 9} 8953$ interface and determine water orientation in the hydration layer of ice, previously (Smit Wilbert and Bakker Huib, 2017). Work is ongoing to characterize AFP on an ice surface and initial datae are promising enough to support water-cage mediated ice adsorption by AFP.

\section{Acknowledgment}

Work on antifreeze proteins is funded by the Department of Science and Technology SERB grant EMR/2016/001333. Authors also gratefully acknowledge the central supercomputing facility (CRAY) at Indian Association for the Cultivation of Science, Kolkata.

Celik Y, Drori R, Pertaya-Braun N, Altan A, Barton T, Bar-Dolev M, Groisman A, Davies P L and Braslavsky I (2013) Microfluidic experiments reveal that antifreeze proteins bound to ice crystals suffice to prevent their growth Proceedings of the National Academy of Sciences 1101309 1314

Chakraborty S and Jana B (2017a) Conformational and hydration properties modulate ice recognition by type I antifreeze protein and its mutants Physical Chemistry Chemical Physics 19 11678-11689

Chakraborty S and Jana B (2017b) Molecular Insight into the Adsorption of Spruce Budworm Antifreeze Protein to an Ice Surface: A Clathrate-Mediated Recognition Mechanism Langmuir 33 7202-7214

Chakraborty S and Jana B (2018) Optimum Number of Anchored Clathrate Water and Its Instantaneous Fluctuations Dictate Ice Plane Recognition Specificities of Insect Antifreeze Protein The Journal of Physical Chemistry B 122 30563067

Chaves D F, Campelo I S, Silva M M A S, Bhat M H, Teixeira D I A, Melo L M, Souza-Fabjan J M G, Mermillod P and Freitas V J F (2016) The use of antifreeze protein type III for vitrification of in vitro matured bovine oocytes Cryobiology 73 324-328

Chen L, DeVries A L and Cheng C-H C (1997) Evolution of antifreeze glycoprotein gene from a trypsinogen gene in Antarctic notothenioid fish Proceedings of the National Academy of Sciences 94 3811-3816

Cheng A and Merz K M (1997) Ice-binding mechanism of winter flounder antifreeze proteins Biophysical Journal 73 28512873 
Cheng C-H C (1998) Evolution of the diverse antifreeze proteins Current Opinion in Genetics \& Development 8 715-720

Cheng C C and DeVries A L (1991) The Role of Antifreeze Glycopeptides and Peptides in the Freezing Avoidance of Cold-Water Fish. In: di Prisco G Life Under Extreme Conditions. Springer Berlin Heidelberg, Berlin, Heidelberg, $1-14$

Congdon T, Notman R and Gibson M I (2013) Antifreeze (Glyco)protein Mimetic Behavior of Poly(vinyl alcohol): Detailed Structure Ice Recrystallization Inhibition Activity Study Biomacromolecules 14 1578-1586

Congdon T R, Notman R and Gibson M I (2016) Influence of Block Copolymerization on the Antifreeze Protein Mimetic Ice Recrystallization Inhibition Activity of Poly(vinyl alcohol) Biomacromolecules 17 3033-3039

DeLuca C I, Davies P L, Ye Q and Jia Z (1998) The effects of steric mutations on the structure of type III antifreeze protein and its interaction with ice1 Journal of Molecular Biology 275 515-525

Deng G, Andrews D W and Laursen R A (1998) Amino acid sequence of a new type of antifreeze protein, from the longhorn sculpin Myoxocephalus octodecimspinosis FEBS Letters $\mathbf{4 0 2}$ 17-20

Devries A L (1971) Glycoproteins as Biological Antifreeze Agents in Antarctic Fishes Science 172 1152-1155

Devries A L and Lin Y (1977) Structure of a peptide antifreeze and mechanism of adsorption to ice Biochimica et Biophysica Acta (BBA) - Protein Structure 495 388-392

DeVries A L and Wohlschlag D E (1969) Freezing Resistance in Some Antarctic Fishes Science 163 1073-1075

Drori R, Celik Y, Davies P L and Braslavsky I (2014) Ice-binding proteins that accumulate on different ice crystal planes produce distinct thermal hysteresis dynamics Journal of The Royal Society Interface 11

Duman J G (1994) Purification and characterization of a thermal hysteresis protein from a plant, the bittersweet nightshade Solanum dulcamara Biochimica et Biophysica Acta (BBA) Protein Structure and Molecular Enzymology 1206 129135

Duman J G (2001) Antifreeze and Ice Nucleator Proteins in Terrestrial Arthropods Annual Review of Physiology 63 327-357

Ebbinghaus S, Meister K, Born B, DeVries A L, Gruebele M and Havenith M (2010) Antifreeze Glycoprotein Activity Correlates with Long-Range Protein"Water Dynamics Journal of the American Chemical Society 132 12210-12211

Ewart K V, Lin Q and Hew C L (1999) Structure, function and evolution of antifreeze proteins Cellular and Molecular

\section{Life Sciences CMLS 55 271-283}

Fei Y, Sun L, Huang T, Shu N, Gao S and Jian L (1994) Isolation and identification of antifreeze protein with high activity in Ammopiptanthus mongolicus Acta Botanica Sinica 36 649-650

Garnham C P, Campbell R L and Davies P L (2011) Anchored clathrate waters bind antifreeze proteins to ice Proceedings of the National Academy of Sciences 108 7363-7367

Garnham C P, Gilbert J A, Hartman C P, Campbell R L, Laybourn-Parry J and Davies PL (2008) $\mathrm{A} \mathrm{Ca}^{2+}$-dependent bacterial antifreeze protein domain has a novel ${ }^{2}$-helical ice-binding fold Biochemical Journal 411171

Gauthier S Y, Scotter A J, Lin F-H, Baardsnes J, Fletcher G L and Davies P L (2008) A re-evaluation of the role of type IV antifreeze protein Cryobiology 57 292-296

Gilbert J A, Hill P J, Dodd C E R and Laybourn-Parry J (2004) Demonstration of antifreeze protein activity in Antarctic lake bacteria Microbiology 150 171-180

Gordon M S, Amdur B H and Scholander P F (1962) FREEZING RESISTANCE IN SOME NORTHERN FISHES The Biological Bulletin 122 52-62

Graether S P, Kuiper M J, Gagne S M, Walker V K, Jia Z, Sykes B D and Davies P L (2000) [beta]-Helix structure and icebinding properties of a hyperactive antifreeze protein from an insect Nature 406 325-328

Griffith M, Antikainen M, Hon W-C, Pihakaski-Maunsbach K, Yu X-M, Chun J U and Yang D S C (1997) Antifreeze proteins in winter rye Physiologia Plantarum 100 327332

Gronwald W, Loewen M C, Lix B, Daugulis A J, Sönnichsen F D, Davies P L and Sykes B D (1998) The Solution Structure of Type II Antifreeze Protein Reveals a New Member of the Lectin Family Biochemistry 37 4712-4721

Gupta R and Deswal R (2012) Low Temperature Stress Modulated Secretome Analysis and Purification of Antifreeze Protein from Hippophae rhamnoides, a Himalayan Wonder Plant Journal of Proteome Research 11 2684-2696

Haas J, Roth S, Arnold K, Kiefer F, Schmidt T, Bordoli L and Schwede T (2013) The Protein Model Portal-a comprehensive resource for protein structure and model information Database 2013 bat031-bat031

Hakim A, Nguyen J B, Basu K, Zhu D F, Thakral D, Davies P L, Isaacs F J, Modis Y and Meng W (2013) Crystal Structure of an Insect Antifreeze Protein and Its Implications for Ice Binding Journal of Biological Chemistry 288 12295-12304

Halder R and Jana B (2018) Unravelling the CompositionDependent Anomalies of Pair Hydrophobicity in Water- 
Ethanol Binary Mixtures The Journal of Physical Chemistry B 122 6801-6809

Hanada Y, Nishimiya Y, Miura A, Tsuda S and Kondo H (2014) Hyperactive antifreeze protein from an Antarctic sea ice bacterium Colwellia sp. has a compound ice-binding site without repetitive sequences FEBS Journal 281 35763590

Hayward J A and Haymet A D J (2001) The ice/water interface: Molecular dynamics simulations of the basal, prism, $\{2021\}$, and $\{2110\}$ interfaces of ice Ih The Journal of Chemical Physics 114 3713-3726

Hon W C, Griffith M, Mlynarz A, Kwok Y C and Yang D S C (1995) Antifreeze Proteins in Winter Rye Are Similar to Pathogenesis-Related Proteins Plant Physiology 109879

Jia Z and Davies P L (2002) Antifreeze proteins: An unusual receptor-ligand interaction Trends in Biochemical Sciences 27 101-106

Jia Z, DeLuca C I, Chao H and Davies P L (1996) Structural basis for the binding of a globular antifreeze protein to ice Nature 384 285-288

Jo J W, Jee B C, Suh C S and Kim S H (2012) The Beneficial Effects of Antifreeze Proteins in the Vitrification of Immature Mouse Oocytes PLOS ONE 7 e37043

Karim O A and Haymet A D J (1988) The ice/water interface: A molecular dynamics simulation study The Journal of Chemical Physics 89 6889-6896

Kim M, Gwak Y, Jung W and Jin E (2017) Identification and Characterization of an Isoform Antifreeze Protein from the Antarctic Marine Diatom, Chaetoceros neogracile and Suggestion of the Core Region Marine Drugs 15

Knight C A, Cheng C C and DeVries A L (1991) Adsorption of alpha-helical antifreeze peptides on specific ice crystal surface planes Biophysical Journal 59 409-418

Knight C A, De Vries A L and Oolman L D (1984) Fish antifreeze protein and the freezing and recrystallization of ice Nature 308 295-296

Knight C A, Hallett J and DeVries A L (1988) Solute effects on ice recrystallization: An assessment technique Cryobiology 25 55-60

Kristiansen E and Zachariassen K E (2005) The mechanism by which fish antifreeze proteins cause thermal hysteresis Cryobiology 51 262-280

Kuiper M J, Davies P L and Walker V K (2001) A Theoretical Model of a Plant Antifreeze Protein from Lolium perenne Biophysical Journal 81 3560-3565

Kun H and Mastai Y (2007) Activity of short segments of Type I antifreeze protein Peptide Science $\mathbf{8 8}$ 807-814
Laezza A, Casillo A, Cosconati S, Biggs C I, Fabozzi A, Paduano L, Iadonisi A, Novellino E, Gibson M I, Randazzo A, Corsaro M M and Bedini E (2017) Decoration of Chondroitin Polysaccharide with Threonine: Synthesis, Conformational Study, and Ice-Recrystallization Inhibition Activity Biomacromolecules 18 2267-2276

Lee J, Kim S K, Youm H W, Kim H J, Lee J R, Suh C S and Kim S H (2015) Effects of Three Different Types of Antifreeze Proteins on Mouse Ovarian Tissue Cryopreservation and Transplantation PLOS ONE 10 e0126252

Lee J K, Park K S, Park S, Park H, Song Y H, Kang S H and Kim $\mathrm{H} \mathrm{J}$ (2010) An extracellular ice-binding glycoprotein from an Arctic psychrophilic yeast Cryobiology 60 222-228

Li Q Z, Yeh Y, Liu J J, Feeney R E and Krishnan V V (2006) A two-dimensional adsorption kinetic model for thermal hysteresis activity in antifreeze proteins The Journal of Chemical Physics 124204702

Liu S, Wang W, von Moos E, Jackman J, Mealing G, Monette R and Ben R N (2007a) In Vitro Studies of Antifreeze Glycoprotein (AFGP) and a C-Linked AFGP Analogue Biomacromolecules 8 1456-1462

Liu Y, Li Z, Lin Q, Kosinski J, Seetharaman J, Bujnicki J M, Sivaraman J and Hew C-L (2007b) Structure and Evolutionary Origin of Ca2+-Dependent Herring Type II Antifreeze Protein PLOS ONE 2 e548

MacDonald M J, Cornejo N R and Gellman S H (2017) Inhibition of Ice Recrystallization by Nylon-3 Polymers ACS Macro Letters 6 695-699

Madura J D, Wierzbicki A, Harrington J P, Maughon R H, Raymond J A and Sikes C S (1994) Interactions of the Dand L-Forms of Winter Flounder Antifreeze Peptide with the $\{201\}$ Planes of Ice Journal of the American Chemical Society $116417-418$

Mallajosyula S S, Vanommeslaeghe K and MacKerell AD (2014) Perturbation of Long-Range Water Dynamics as the Mechanism for the Antifreeze Activity of Antifreeze Glycoprotein The Journal of Physical Chemistry B 118 11696-11706

Meister K, Ebbinghaus S, Xu Y, Duman J G, DeVries A, Gruebele M, Leitner D M and Havenith M (2013) Long-range protein-water dynamics in hyperactive insect antifreeze proteins Proceedings of the National Academy of Sciences $1101617-1622$

Meister K, Strazdaite S, DeVries A L, Lotze S, Olijve L L C, Voets I K and Bakker H J (2014) Observation of ice-like water layers at an aqueous protein surface Proceedings of the National Academy of Sciences 111 17732-17736 
Midya U S and Bandyopadhyay S (2014) Hydration Behavior at the Ice-Binding Surface of the Tenebrio molitor Antifreeze Protein The Journal of Physical Chemistry B 118 47434752

Midya U S and Bandyopadhyay S (2017) Interfacial Water Arrangement in the Ice-Bound State of an Antifreeze Protein: A Molecular Dynamics Simulation Study Langmuir

Nutt D R and Smith J C (2008) Dual Function of the Hydration Layer around an Antifreeze Protein Revealed by Atomistic Molecular Dynamics Simulations Journal of the American Chemical Society 130 13066-13073

Olijve L L C, Hendrix M M R M and Voets I K (2016a) Influence of Polymer Chain Architecture of Poly(vinyl alcohol) on the Inhibition of Ice Recrystallization Macromolecular Chemistry and Physics 217 951-958

Olijve L L C, Meister K, DeVries AL, Duman J G, Guo S, Bakker $\mathrm{H} \mathrm{J}$ and Voets I K (2016b) Blocking rapid ice crystal growth through nonbasal plane adsorption of antifreeze proteins Proceedings of the National Academy of Sciences 1133740 3745

Parui S and Jana B (2017) Pairwise Hydrophobicity at Low Temperature: Appearance of a Stable Second SolventSeparated Minimum with Possible Implication in Cold Denaturation The Journal of Physical Chemistry B 121 7016-7026

Parui S and Jana B (2018) Molecular Insights into the Unusual Structure of an Antifreeze Protein with a Hydrated Core The Journal of Physical Chemistry B 122 9827-9839

Pertaya N, Marshall C B, Celik Y, Davies P L and Braslavsky I (2008) Direct Visualization of Spruce Budworm Antifreeze Protein Interacting with Ice Crystals: Basal Plane Affinity Confers Hyperactivity Biophysical Journal 95 333-341

Raymond J A and DeVries A L (1977) Adsorption inhibition as a mechanism of freezing resistance in polar fishes Proceedings of the National Academy of Sciences 742589 2593

Robles V, Barbosa V, Herráez M P, Martínez-Páramo S and Cancela M L (2007) The antifreeze protein type I (AFP I) increases seabream (Sparus aurata) embryos tolerance to low temperatures Theriogenology 68 284-289

Samanta T and Bagchi B (2018) Temperature effects on the hydrophobic force between two graphene-like surfaces in liquid water Journal of Chemical Sciences 13029

Samanta T, Biswas R, Banerjee S and Bagchi B (2018) Study of distance dependence of hydrophobic force between two graphene-like walls and a signature of pressure induced structure formation in the confined water The Journal of Chemical Physics 149044502

Scotter A J, Marshall C B, Graham L A, Gilbert J A, Garnham C $\mathrm{P}$ and Davies P L (2006) The basis for hyperactivity of antifreeze proteins Cryobiology 53 229-239

Simpson D J, Smallwood M, Twigg S, Doucet C J, Ross J and Bowles D J (2005) Purification and characterisation of an antifreeze protein from Forsythia suspensa (L.) Cryobiology 51 230-234

Smit Wilbert J and Bakker Huib J (2017) The Surface of Ice Is Like Supercooled Liquid Water Angewandte Chemie International Edition 56 15540-15544

Smolin N and Daggett V (2008) Formation of Ice-like Water Structure on the Surface of an Antifreeze Protein The Journal of Physical Chemistry B 112 6193-6202

Sun T, Lin F-H, Campbell R L, Allingham J S and Davies P L (2014) An Antifreeze Protein Folds with an Interior Network of More Than 400 Semi-Clathrate Waters Science 343 795-798

Takamichi M, Nishimiya Y, Miura A and Tsuda S (2007) Effect of annealing time of an ice crystal on the activity of type III antifreeze protein The FEBS Journal 274 6469-6476

Todde G, Hovmöller S and Laaksonen A (2015) Influence of Antifreeze Proteins on the Ice/Water Interface The Journal of Physical Chemistry B 119 3407-3413

Todde G, Whitman C, Hovmöller S and Laaksonen A (2014) Induced Ice Melting by the Snow Flea Antifreeze Protein from Molecular Dynamics Simulations The Journal of Physical Chemistry B 118 13527-13534

Tomczak M M, Marshall C B, Gilbert J A and Davies P L (2003) A facile method for determining ice recrystallization inhibition by antifreeze proteins Biochemical and Biophysical Research Communications 311 1041-1046

Wen D and Laursen R A (1992a) A model for binding of an antifreeze polypeptide to ice Biophysical Journal 63 16591662

Wen D and Laursen R A (1992b) Structure-function relationships in an antifreeze polypeptide. The role of neutral, polar amino acids Journal of Biological Chemistry 267 1410214108

Wierzbicki A, Dalal P, Cheatham Iii T E, Knickelbein J E, Haymet A D J and Madura J D (2007) Antifreeze Proteins at the Ice/Water Interface: Three Calculated Discriminating Properties for Orientation of Type I Proteins Biophysical Journal 93 1442-1451

Wilkinson B L, Stone R S, Capicciotti C J, Thaysen-Andersen M, Matthews J M, Packer N H, Ben R N and Payne R J 
(2012) Total Synthesis of Homogeneous Antifreeze Glycopeptides and Glycoproteins Angewandte Chemie 124 3666-3670

Xiao N, Suzuki K, Nishimiya Y, Kondo H, Miura A, Tsuda S and Hoshino T (2009) Comparison of functional properties of two fungal antifreeze proteins from Antarctomyces psychrotrophicus and Typhula ishikariensis The FEBS Journal 277 394-403

Yang D S C, Hon W-C, Bubanko S, Xue Y, Seetharaman J, Hew C $\mathrm{L}$ and Sicheri F (1998) Identification of the Ice-Binding Surface on a Type III Antifreeze Protein with a "Flatness Function” Algorithm Biophysical Journal 74 2142-2151
Younis Abdelmoneem I, Rooks B, Khan S and Gould Kenneth G (2013) The Effects of Antifreeze Peptide III (AFP) and Insulin Transferrin Selenium (ITS) on Cryopreservation of Chimpanzee (Pan troglodytes) Spermatozoa Journal of Andrology 19 207-214

Zhang W and Laursen R A (1998) Structure-Function Relationships in a Type I Antifreeze Polypeptide: THE ROLE OF THREONINE METHYL AND HYDROXYL GROUPS IN ANTIFREEZE ACTIVITY Journal of Biological Chemistry 273 34806-34812. 